\title{
Supernova forecast with strong lensing
}

\author{
Yudai Suwa ${ }^{1 \star}$ \\ ${ }^{1}$ Center for Gravitational Physics, Yukawa Institute for Theoretical Physics, Kyoto University, Kyoto, 606-8502, Japan
}

Accepted. Received.

\begin{abstract}
In the coming LSST era, we will observe $\mathcal{O}(100)$ of lensed supernovae (SNe). In this paper, we investigate possibility for predicting time and sky position of a supernova using strong lensing. We find that it will be possible to predict the time and position of the fourth image of SNe which produce four images by strong lensing, with combined information from the three previous images. It is useful to perform multi-messenger observations of the very early phase of supernova explosions including the shock breakout.
\end{abstract}

Key words: gravitational lensing: strong — supernovae: general

\section{INTRODUCTION}

Supernovae (SNe) represent the final phase of a massive star. When a shock that forms inside the star pierces the stellar surface, so called shock breakout (SBO) takes place, which makes a bright flush in X-ray/ultraviolet bands (see Waxman \& Katz 2016, for a recent review). SBO and the early light curve (LC) of SN are useful probes to investigate the very final stage of stellar evolution. There have been three (including candidate) observations of SBO so far (SN2008D, Soderberg et al. 2008; SNLS-04D2dc, Schawinski et al. 2008; KSN 2011d, Garnavich et al. 2016). SN2008D was serendipitously observed by Swift, and others were found by survey programs.

The biggest survey volume in optical/infrared bands is achieved by Subaru Hyper Suprime-Cam (Miyazaki et al. 2012) at the current moment and will be made by the Large Synoptic Survey Telescope (LSST) (LSST Science Collaboration et al. 2017) in the near future. There are also other survey programs, such as the Palomar Transient Factory (PTF, Law et al. 2009), Panoramic Survey Telescope and Rapid Response System (Pan-STARRS1, Kaiser et al. 2010), the High Cadence Transient Survey (HITS, Förster et al. 2016), the Kiso Supernova Survey (KISS, Morokuma et al. 2014), etc. These surveys will eventually detect SBO soon. Follow-up observations are possible only after the alert sent by these survey programs, so that multi-telescope observations of SBO is very difficult even with these magnificent survey programs. Accordingly, we propose a different way to catch SBO using strong lensing.

After more than 50 years since the original idea by Refsdal (1964), there have been three observations of strongly lensed SNe. The first one was PS1-10afx, which is a Type Ia

* E-mail: suwa@yukawa.kyoto-u.ac.jp
SN. This event was not detected as resolved multiple images, but it was magnified by a factor of 30 because of strong lensing (Quimby et al. 2013, 2014). The next one was SN Refsdal, which is an SN 1987-like core collapse SN. This was the first ever lensed SN with resolved multiple images (Kelly et al. 2015). Importantly, from the positions of these images, it was predicted that there would be an additional image one year after the discovery of them and it indeed appeared (Kelly et al. 2016). This is the first $S N$ forecast. The third one was iPTF16geu, which is a Type Ia SN. At first, multiple images were not resolved by PTF, but follow-up observations by Hubble Space Telescope (HST) resolved multiple images, so that this is the first lensed Type Ia SN with resolved multiple images (Goobar et al. 2017). It clearly demonstrated the usefulness of observations of multi-telescope for lensed SNe.

Here, we mention SN Refsdal a little bit more in detail. This is an SN occurring at $z=1.49$ and lensed by galaxy cluster at $z=0.54$. The SN itself was lensed by a galaxy between host galaxy and observer, and found as four images around host galaxy. Furthermore, the SN host galaxy was also lensed by the cluster, producing three images. Followup analysis suggested that two more images were possible, in addition to the already observed four images (e.g. Oguri 2015; Treu et al. 2016). One of them would have appeared 17 years ago, while another one would appear one year after the original four images. This case allowed a stringent test of strong lensing models. Indeed, a new image was found at the predicted time and sky location.

It was predicted that LSST will observe more than 100 strongly lensed SNe (Oguri \& Marshall 2010). This is actually a conservative estimate and the number can be even more than 1000, depending on the criteria (M. Oguri, private communication). In this work, we will demonstrate the 
viability of SN forecast and multi-messenger observations of $\mathrm{SBO}$, using lensed SNe.

\section{TIME DELAY, MAGNITUDE, AND SEPARATION}

In this work, we specify LSST for triggering new SNe. LSST is a survey program, which uses a telescope with $8.4 \mathrm{~m}$ mirror, 15 s exposure, $9.6 \mathrm{deg}^{2}$ of field of view, and six bands. It sweeps whole sky with $\approx 24$ mag of $5 \sigma$ depth, a typical seeing full width at half-maximum (FWHM) being 0.75 arcsec, and 5 day cadence. A number of transient astrophysical objects will be detected, for instance, $\mathcal{O}\left(10^{6}\right)$ of SNe, during its ten-year survey. A predicted number of lensed SN, which will be observed by LSST is $\sim 130$ (Oguri \& Marshall 2010). Among the lensed SNe, the fraction of Type Ia is $\sim 34 \%,{ }^{1}$ $\mathrm{Ib} / \mathrm{c}$ is $\sim 31 \%$, IIL is $\sim 5 \%$, IIP is $\sim 15 \%$, and IIn is $\sim 15 \%$. In this work we focus on Type $\mathrm{Ib} / \mathrm{c} \mathrm{SNe}$, since they have a relatively short timescale in $\mathrm{LC}$ evolution because of their small ejecta mass, and hence they make the systematic error of the SBO time estimation determined from the LC smaller than for other SN types.

Oguri \& Marshall (2010) conducted Monte-Carlo calculations regarding quasar and SN lensing, and estimated numbers of predicted lensed transient objects for various survey programs. Since they made their mock data public, ${ }^{2}$ we use their data in this work. In their calculations, they took into account not only limiting magnitudes, but also angular resolutions to resolve multiple images. They omitted images with separation being smaller than $2 / 3$ of the seeing FWHM of the survey programs (seeing FWHM being 0.75 arcsec for LSST). After their prediction, iPTF16geu was observed without resolving multiple images by the initial survey telescope (PTF), but it was resolved into multiple images by a follow-up observation done by HST. Therefore, the number of lensed objects resolved into multiple images can be significantly larger than their original estimate, i.e. their estimate is rather conservative.

In the following, we investigate lensed Type Ib/c SNe in mock catalog by Oguri \& Marshall (2010). Their mock sample limits events whose third brightest images being brighter than $22.6 \mathrm{mag}$, which is $0.7 \mathrm{mag}$ brighter than the magnitude limit of each visit (23.3 mag for LSST). Table 1 summaries the properties. Among their 1219 lensed SN samples, which are ten times oversampled, we select 376 Type Ib/c SNe. 86 of them have four images, so that in the LSST era we will observe roughly one SN with four images every year. It should be noted that the mean peak magnitude $\left\langle m_{\text {lens }}\right\rangle$ does not depend on the number of images.

The top panel of Figure 1 presents the peak magnitudes of the second, third, and fourth images as a function of delay time from the first image, for lensed SNe with four images. As is seen from the figure, longer delay time leads to a dimmer image. In some systems, these fourth images appear $\mathcal{O}(10)$ days after the first image emerged with magnitude

1 The number is slightly different from value in Table 3 of Oguri \& Marshall (2010), because of Poisson noise of sampling.

2 Since it was not available online when the author performed this work, we asked M. Oguri to provide it.
Table 1. Summary of mock Type Ib/c SN data from Oguri \& Marshall (2010). The first half gives characteristics of all events, and the second half gives events having four images. $\langle\cdots\rangle$ gives mean value with the standard deviation. $z_{s}$ is source redshift, $z_{l}$ is lens redshift, $m_{\mathrm{org}}$ is original peak apparent magnitude without lensing, $m_{\text {lens }}$ is peak apparent magnitude with lensing, $\theta_{\text {lens }}$ is separation from the first image, and $t_{\text {delay }}$ is the time delay of the fourth image from the first image.

\begin{tabular}{ll}
\hline all lensed Type Ib/c SNe & \\
\hline number of SNe & 376 \\
$\left\langle z_{s}\right\rangle$ & $0.789 \pm 0.231$ \\
$\left\langle z_{l}\right\rangle$ & $0.326 \pm 0.166$ \\
$\left\langle m_{\text {org }}\right\rangle$ & $22.6 \pm 1.02$ \\
number of images & 926 \\
$\left\langle m_{\text {lens }}\right\rangle$ & $21.6 \pm 1.00$ \\
\hline lensed Type Ib/c SNe with 4 images \\
\hline number of SNe \\
$\left\langle m_{\text {lens }}\right\rangle$ \\
$\left\langle\theta_{\text {lens }}\right\rangle(\operatorname{arcsec})^{a}$ & 86 \\
$\left\langle\log _{10}\left(t_{\text {delay }} / \text { day }\right)\right\rangle^{b}$ & $21.7 \pm 1.18$ \\
\hline
\end{tabular}

${ }^{a}$ Mean separation from the first image, for second, third and fourth images.

${ }^{b}$ Mean logarithmic time delay between the first and fourth images.

brighter than 24 , which can be observed by $2-4$ m-size telescopes. The bottom panel of the figure indicates the angular separation of images from the first image. It shows a large scatter and no apparent correlation with time delay. Typical separations are about 1 arcsec, which can be resolved by ground-based telescopes.

Figure 2 presents an example LC of a lensed Type Ib/c $\mathrm{SN}$. The lens galaxy ID in the mock data is 7738038 , which has $z_{s}=0.87$ and $z_{l}=0.314$. The apparent peak magnitude in I-band without lensing is $22.5 \mathrm{mag}$. Since Oguri \& Marshall (2010) only took into account the peak magnitude, we construct the LC by using a typical Type Ib/c SN, SN1999ex (Stritzinger et al. 2002). In the figure, the limiting magnitude of LSST per visit $(10 \sigma)$ is shown as a grey horizontal line. One can see that the first image can be observed about 30 days before its peak and the second image will appear above the detection threshold 18 days after the first image emergence. The third and fourth ones will be found about 28 and 58 days after the first image. In this case, the SBO emission of the fourth image will be observed after the emergence of the third image.

\section{STRATEGY}

In this section, we discuss a strategy to perform a multimessenger observation campaign for SBO of fourth images from lensed SNe. An ideal scenario is as follows:

(i) Find a new SN by LSST survey.

(ii) A second image appears $\mathcal{O}(1) \mathrm{d}$ after the first image.

(iii) One calculates the lens potential based on these two images and predict the position and time of the third image.

(iv) By observing the third image with deeper and more frequent observations, one calibrates the lens potential 

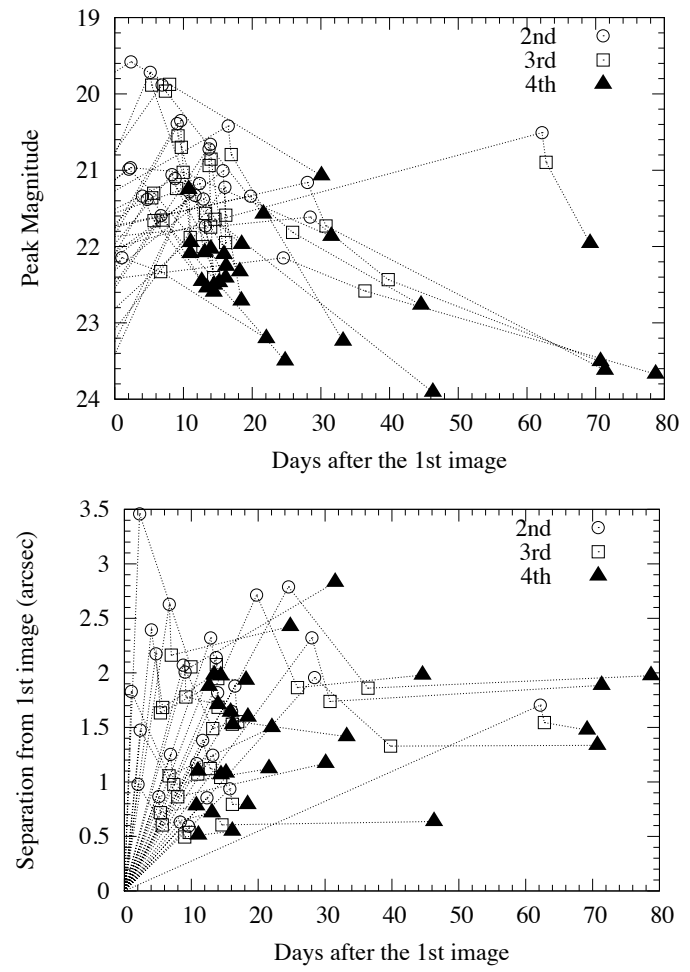

Figure 1. (Top) Peak magnitude of each image as a function of time delay from the first image. Among Type Ib/c SNe given by Oguri \& Marshall (2010), 28 events, which produce third images later than five days and fourth images later than ten days but no later than a hundred days after the first image, are shown. Open circles, open squares, and closed triangles indicate second, third and fourth images, respectively. Dashed lines connect images from the same SNe. (Bottom) The same as top panel, but for separation from the first image.

model and LC evolution, and predicts the fourth image more precisely than the third one.

(v) One targets the SBO of the fourth image using multiple telescopes.

In Figure 3, we show our flowchart of the event selection. In this figure, the first and second images should be detected by LSST, and other telescopes are able to conduct more frequent observations for third and fourth images. In order to observe the SBO, the most important part is the time precision of the fourth image. If we can reduce the error, by intensively observing the third image, up to $\lesssim 1 \mathrm{~d}$, the feasibility of the SBO observation becomes remarkably high. For predicting the third image properties, we need to determine at least five lens model parameters if we employ the singular isothermal ellipsoid (SIE), which is used most frequently to model lensing galaxies, assuming redshifts are determined well by photometric data: velocity dispersion; ellipticity; orientation of the lens galaxy; source position. Up to six degrees of freedom can be fixed by observation of the first two images: two image positions; flux ratio; time delay. Hence, in principle, the prediction is possible. Of course, for a more realistic lens model, there are more parameters to be determined. But on the other hand, the observation of the third image does provide significantly more information since, together with the first and second image, there are

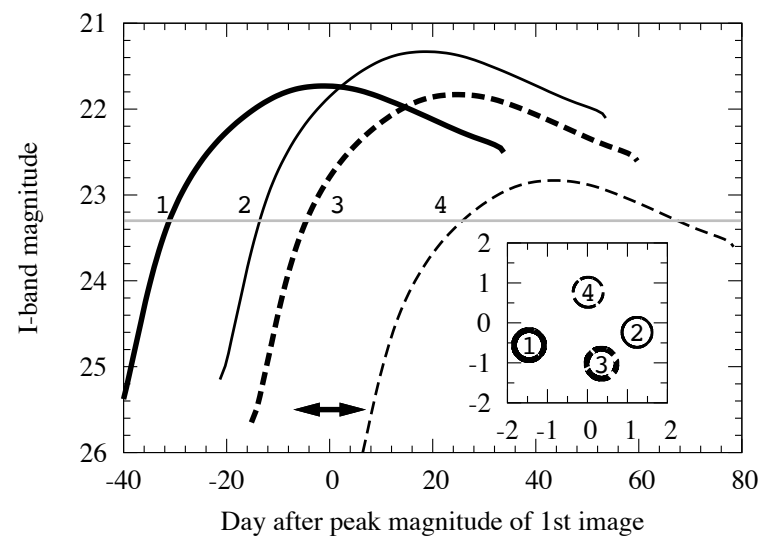

Figure 2. An example light curve in i-band of an event with $z_{s}=0.87$ and $z_{l}=0.314$. Light curve shape is taken from a typical Type Ib/c SN, SN1999ex. First, second, third, and fourth images correspond to thick-solid, thin-solid, thick-dashed, and thindashed lines, respectively. Time delays from the first image are 19.8 (second), 25.9 (third), and 44.6 (fourth) days. The arrow indicates the time of SBO of the fourth image, which is bright in $\mathrm{UV} / \mathrm{X}$-ray bands for the case of a Type Ib/c SN. Horizontal grey line indicates limiting magnitude of LSST. In the small panel, spacial positions of images are shown with a typical seeing FWHM of LSST (0.75 arcsec). Numbers in circles present corresponding image in LC.

now twelve observables: three image positions; three flux ratios; three time delays. It should also be noted that we are neglecting host galaxy distortion, which would provide additional information, so that our results in the following are conservative.

By using the same mock data as Figure 2, we attempt to predict the time delay and position of the fourth image from information of the previous three images with glafic (Oguri 2010), which is a public software package for analyzing gravitational lensing (see Appendix A). A similar study was done in Oguri et al. (2003), but it was more interested in cosmological applications. As input data, we employ sky positions of three images, the redshift of host galaxy, flux ratios, and time delays with respect to the first image, with $1 \sigma$ errors of 0.75 arcsec, $0.5,50 \%$, and 5 days, respectively. The best fit is obtained with the delay time for the fourth image being 44.74 days, which is slightly ( 0.17 day) later than the correct value. ${ }^{3}$ Smaller error values lead to better prediction.

Note that our assumptions of uncertainties are relatively conservative, that is, the time delay error of actual observations could be smaller than the current estimate. The uncertainty of time delays for second and third images ( 5 days here), however, might be longer. In order to check their dependences, we perform the same analysis by changing the uncertainty of the time delay, for instance 3 days or 10 days, and find that the prediction changes only $\mathcal{O}(0.01)$ day. Namely, the uncertainty of the time delay is not essen-

3 The same analysis for the third image using information of the first and the second images indicates that the calculated time delay is 4.0 days earlier than correct value. Thus, the prediction of the third image is not very precise, but still feasible. 


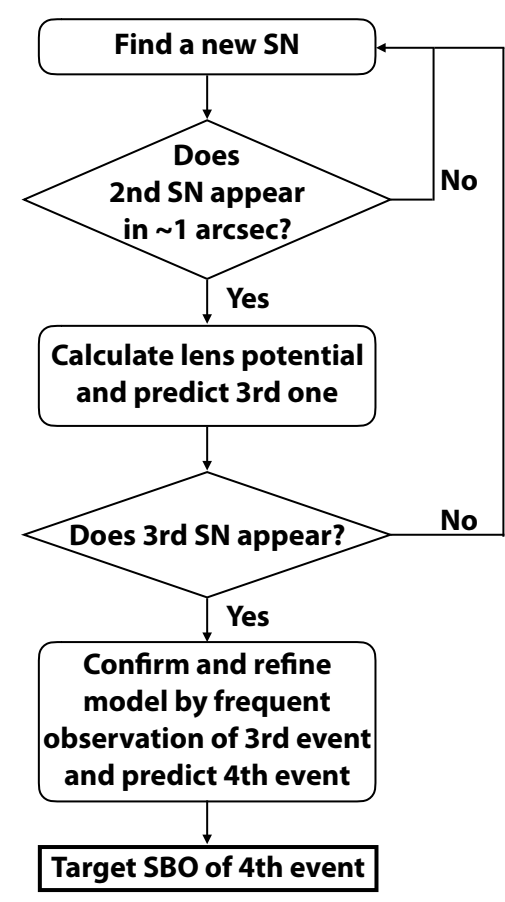

Figure 3. A flowchart of the event selection. First and second images are found by LSST and third and fourth images are observed by other telescopes.

tial for the prediction of the fourth image for this particular case.

We also conduct the same analysis for other mock samples of Oguri \& Marshall (2010). For this analysis, we use the following two criteria: i) time delay between the first and the third images is longer than five days; ii) time delay between the first and the fourth images is longer than ten days. Then, we obtain 29 events from the mock data. Among them, 23 events $(79 \%)$ present good agreements between the estimated time delays and the correct values within one day. Other six events indicate small image separations $(\sim 0.1$ $\operatorname{arcsec}$ ) or short time delays between the first and the third images ( $\sim 5$ days). By performing the analysis with smaller position and time delay uncertainties than the previous one, i.e. 0.3 arcsec and 1 day, we find that the prediction error is largely improved (within one day) for five of them. One exceptional case (ID 69154345) has the considerably delayed fourth image which appears 140.7 days after the first image. Therefore, after the detection of the third image, the prediction of fourth image is doable in most cases. Note that this result may change with different assumption about the lens model, so that a more systematic study is needed and will be presented in following works.

For the current purpose, the fourth image needs to appear later than 10 days after the first image, in order to complete these analyses before the fourth image emerges. In the mock catalog from Oguri \& Marshall (2010), there are 50 Type Ib/c SNe, which have $t_{\text {delay }}$ (delay time between the first and fourth images) $>10$ days and 24 SNe with $t_{\text {delay }}>20$ days. Note that their catalog is based on the rather stringent criterion that multiple images be detected with more than $10 \sigma$ and be resolved by LSST alone. By using other telescopes, which have better sensitivity and angular resolution, the event rate can be increased by a factor of 10 . Then, we may observe $\sim 1$ of these events per year in the LSST era (see also Goldstein \& Nugent 2017).

\section{SUMMARY AND DISCUSSION}

In the coming LSST era, we will observe $\mathcal{O}(100)$ of lensed SNe. In this paper, we investigate the feasibility of predicting the emergence of strongly lensed supernovae, based on mock data given by Oguri \& Marshall (2010). Considering a family of lens models generating four images, we found that it will be possible to predict the time and position of the fourth SN image, given observations of the preceding images. In particular, if the separation of images is, roughly speaking, larger than PSF ( 0.75 arcsec in this study) and the time delay between the first and third image is longer than the observation cadence (five days), the prediction of the forth image is possible within one day. The largest systematic error for evaluating the time of SBO is LC modeling of the SN. We can reduce the error by conducting detailed observations of the third image.

The microlensing effects would also introduce some error (Dobler \& Keeton 2006), which is not taken into account in this study. Recently, Goldstein et al. (2017) investigated the microlensing impact on LC of Type Ia SNe, and showed that by making use of multi-band LCs the time delay error by microlensing can be reduced to $\sim 1 \%$ level. A similar study for Type Ib/c SNe is necessary to give uncertainties of the prediction of SBO.

Not only SBO, also the early LC of SNe contains rich information. For instance, with early X-ray emission, the explosion scenario of Type Ia (single degenerate or double degenerate) can be distinguished (Kasen 2010). For corecollapse supernovae, the early LC provides the very final stage of mass ejection from the progenitor stars, which may change the features of SBO. If the SBO takes place in the dense wind, the timescale would become longer (Tanaka et al. 2016). These features can be tackled by multimessenger observations of early LCs.

\section{ACKNOWLEDGEMENTS}

We acknowledge M. Oguri for inspiring this work, providing their mock data, and fruitful discussion. We also thank N. Tominaga for discussing about optical transient observation and M. Werner for insightful comments on the degrees of freedom of lens potential. This study was supported in part by the Grant-in-Aid for Scientific Research (Nos. 16H00869, 16K17665, and 17H02864), MEXT as "Priority Issue on Post-K computer" (Elucidation of the Fundamental Laws and Evolution of the Universe) and JICFuS.

\section{APPENDIX A: GLAFIC}

For estimating delay time of the fourth image, we employ glafic ${ }^{4}$ version 1.2.8 with the following input file.

${ }^{4}$ http://www.slac.stanford.edu/ oguri/glafic/ 


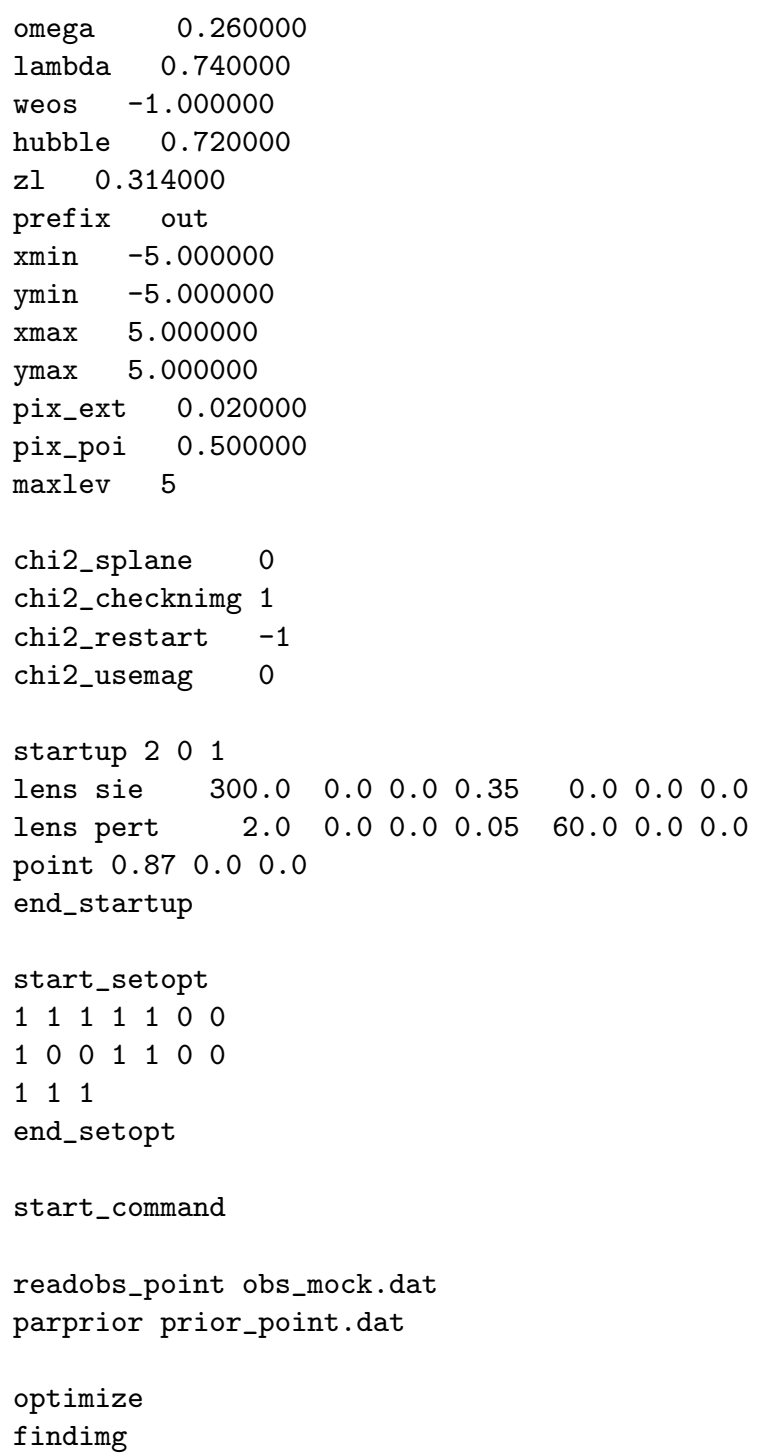

\section{REFERENCES}

Dobler G., Keeton C. R., 2006, ApJ, 653, 1391

Förster F., Maureira J. C., San Martín J., et al., 2016, ApJ, 832, 155

Garnavich P. M., Tucker B. E., Rest A., et al., 2016, ApJ, 820, 23

Goldstein D. A., Nugent P. E., 2017, ApJ, 834, L5

Goldstein, D. A., Nugent, P. E., Kasen, D. N., \& Collett, T. E. 2017, arXiv:1708.00003

Goobar, A., Amanullah, R., Kulkarni, S. R., et al. 2017, Science, 356, 291

Kaiser N., Burgett W., Chambers K., et al., 2010, in Society of Photo-Optical Instrumentation Engineers (SPIE) Conference Series, vol. 7733 of Society of Photo-Optical Instrumentation Engineers (SPIE) Conference Series, 0

Kasen D., 2010, ApJ, 708, 1025

Kelly P. L., Rodney S. A., Treu T., et al., 2015, Science, 347, 1123

Kelly P. L., Rodney S. A., Treu T., et al., 2016, ApJ, 819, L8

Law N. M., Kulkarni S. R., Dekany R. G., et al., 2009, PASP, 121,1395

LSST Science Collaboration, Marshall, P., Anguita, T., et al. 2017, arXiv: 1708.04058

Miyazaki S., Komiyama Y., Nakaya H., et al., 2012, in Society of
Photo-Optical Instrumentation Engineers (SPIE) Conference Series, vol. 8446 of Society of Photo-Optical Instrumentation Engineers (SPIE) Conference Series, 0

Morokuma T., Tominaga N., Tanaka M., et al., 2014, PASJ, 66, 114

Oguri M., 2010, PASJ, 62, 1017

Oguri M., 2015, MNRAS, 449, L86

Oguri M., Marshall P. J., 2010, MNRAS, 405, 2579

Oguri M., Suto Y., Turner E. L., 2003, ApJ, 583, 584

Quimby R. M., Oguri M., More A., et al., 2014, Science, 344, 396 Quimby R. M., Werner M. C., Oguri M., et al., 2013, ApJ, 768, L20

Refsdal S., 1964, MNRAS, 128, 307

Schawinski K., Justham S., Wolf C., et al., 2008, Science, 321, 223

Soderberg A. M., Berger E., Page K. L., et al., 2008, Nature, 453, 469

Stritzinger M., Hamuy M., Suntzeff N. B., et al., 2002, AJ, 124, 2100

Tanaka M., Tominaga N., Morokuma T., et al., 2016, ApJ, 819, 5

Treu T., Brammer G., Diego J. M., et al., 2016, ApJ, 817, 60

Waxman, E., \& Katz, B. 2016, arXiv:1607.01293 